\begin{tabular}{|c|c|}
\hline Title & $\begin{array}{l}\text { Tunnel magnetoresistance in fully epitaxial magnetic tunnel junctions with a full-Heusler alloy thin film of } \\
\text { Co2CrO.6Fe0.4A I and a MgO tunnel barrier }\end{array}$ \\
\hline Author(s) & Marukame, T akao; Y amamoto, Masafumi \\
\hline Citation & $\begin{array}{l}\text { Journal of A pplied Physics, 101(8), } 083906 \\
\text { https://doi.org/10.1063/1.2718284 }\end{array}$ \\
\hline Issue Date & $2007-4-30$ \\
\hline Doc URL & http:/hdl.handle.net/2115/50638 \\
\hline Rights & $\begin{array}{l}\text { Copyright } 2007 \text { A merican Institute of Phy sics. This article may be downloaded for personal use only. A ny other use } \\
\text { requires prior permission of the author and the A merican Institute of Physics. The following article appeared in J. A ppl. } \\
\text { Phys. 101, } 083906 \text { (2007) and may be found at https://dx.doi.org/10.1063/1.2718284 }\end{array}$ \\
\hline Type & article \\
\hline File Information & JAP101_083906.pdf \\
\hline
\end{tabular}

Instructions for use 


\title{
Tunnel magnetoresistance in fully epitaxial magnetic tunnel junctions with a full-Heusler alloy thin film of $\mathrm{CO}_{2} \mathrm{Cr}_{0.6} \mathrm{Fe}_{0.4} \mathrm{Al}$ and a MgO tunnel barrier
}

\author{
Takao Marukame and Masafumi Yamamoto ${ }^{a)}$ \\ Division of Electronics for Informatics, Graduate School of Information Science and Technology, Hokkaido \\ University, N14, W9, Kita-ku, Sapporo 060-0814, Japan
}

(Received 17 January 2007; accepted 13 February 2007; published online 30 April 2007)

\begin{abstract}
Fully epitaxial magnetic tunnel junctions (MTJs) were fabricated with a full-Heusler alloy $\mathrm{Co}_{2} \mathrm{Cr}_{0.6} \mathrm{Fe}_{0.4} \mathrm{Al}$ (CCFA) thin film and a $\mathrm{MgO}$ tunnel barrier. Pseudo-spin-valve-type, fully epitaxial $\mathrm{CCFA} / \mathrm{MgO} / \mathrm{CO}_{50} \mathrm{Fe}_{50}$ MTJs with a CCFA film composition close to the stoichiometric one demonstrated high tunnel magnetoresistance (TMR) ratios of $90 \%$ at room temperature (RT) and $240 \%$ at $4.2 \mathrm{~K}$. These high TMR ratios were attributed to the increased spin polarization at the Fermi level due to the increased ratio of the $B 2$ region to the $A 2$ region in CCFA films achieved by bringing the CCFA film composition close to the stoichiometric one. Fully epitaxial $\mathrm{CCFA} / \mathrm{MgO} / \mathrm{Co}_{50} \mathrm{Fe}_{50}$ MTJs with exchange biasing were also fabricated, where a $\mathrm{Co}_{50} \mathrm{Fe}_{50}$ upper electrode was used in a synthetic ferrimagnetic $\mathrm{Co}_{50} \mathrm{Fe}_{50} / \mathrm{Ru} / \mathrm{Co}_{90} \mathrm{Fe}_{10}$ trilayer exchange-biased with an IrMn layer through the $\mathrm{Co}_{90} \mathrm{Fe}_{10} / \mathrm{IrMn}$ interface. These exchange-biased CCFA/MgO/ $\mathrm{Co}_{50} \mathrm{Fe}_{50}$ MTJs exhibited further enhanced TMR ratios of $109 \%$ at RT and $317 \%$ at 4.2 K. We suggested enhancement of the TMR ratio by a coherent tunneling contribution for fully epitaxial CCFA/MgO/ $\mathrm{Co}_{50} \mathrm{Fe}_{50}$ MTJs. (C) 2007 American Institute of Physics.
\end{abstract}

[DOI: 10.1063/1.2718284]

\section{INTRODUCTION}

In the field of spintronics, where the spin of the electron is employed as an additional degree of freedom in electronics, it is essential to generate highly spin-polarized conduction electrons and transport them. ${ }^{1}$ Half-metallic ferromagnets (HMFs) are characterized by an energy gap at the Fermi level $\left(E_{F}\right)$ for one spin direction, leading to a complete spin polarization at $E_{F}$ (Ref. 2). The potentially high spin polarization of HMFs is highly preferable for spintronic devices.

Co-based full-Heusler alloy thin films have been studied intensively ${ }^{3-12}$ because of the half-metallic ferromagnetic nature theoretically predicted for some of these alloys ${ }^{13,14}$ and because of their high Curie temperatures, which are well above room temperature (RT) (Ref. 15). The potentially high spin polarization of Co-based full-Heusler alloys is greatly advantageous for achieving high tunnel magnetoresistance (TMR) ratios in magnetic tunnel junctions (MTJs) (Refs. 16-33) and high magnetoresistance ratios in giant magnetoresistance devices. $^{34,35}$ It is also advantageous for efficient spin injection from ferromagnetic electrodes into semiconductors ${ }^{36,37}$ and current-induced magnetization switching in MTJs.

One Co-based full-Heusler alloy, in particular, $\mathrm{Co}_{2} \mathrm{Cr}_{0.6} \mathrm{Fe}_{0.4} \mathrm{Al}$ (CCFA), features theoretically predicted high spin polarizations for both the ordered $L 2_{1}$ structure and the disordered $B 2$ one, ${ }^{38}$ along with a relatively high $T_{c}$ around $750 \mathrm{~K} .{ }^{39,40}$ The spin polarization theoretically predicted for the $L 2{ }_{1}$ structure is 0.90 and that for the $B 2$ one is

\footnotetext{
${ }^{a)}$ Author to whom correspondence should be addressed; electronic mail: yamamoto@nano.ist.hokudai.ac.jp
}

$0.78 .^{38}$ The structural and magnetic properties of bulk CCFA (Refs. 39-41) and epitaxially grown CCFA thin films ${ }^{8-10,21,26}$ have been investigated.

Inomata et al. first demonstrated a relatively high TMR ratio of $16 \%$ at RT for MTJs using a Co-based full-Heusler alloy thin film, where a polycrystalline CCFA thin film as a lower electrode and an amorphous $\mathrm{AlO}_{x}$ tunnel barrier were used. ${ }^{16}$ Subsequently, relatively high TMR ratios of up to $70 \%$ at RT have been shown for MTJs using a Co-based full-Heusler alloy thin film- $\mathrm{Co}_{2} \mathrm{MnSi}$ (Ref. 19), $\mathrm{Co}_{2} \mathrm{FeAl}$ (Refs. 20 and 25), or $\mathrm{Co}_{2} \mathrm{MnAl}$ (Ref. 23) - and an amorphous $\mathrm{AlO}_{x}$ tunnel barrier. Sakuraba et al. reported a high TMR ratio of $570 \%$ at $2 \mathrm{~K}(67 \%$ at RT) for MTJs consisting of an epitaxially grown $\mathrm{Co}_{2} \mathrm{MnSi}$ lower electrode, an amorphous $\mathrm{AlO}_{x}$ tunnel barrier, and a highly oriented $\mathrm{Co}_{2} \mathrm{MnSi}$ upper electrode. $^{24}$

We fabricated single-crystal, epitaxially grown $\mathrm{Co}_{2} \mathrm{YZ}$ thin films of CCFA (Refs. 21 and 26), $\mathrm{Co}_{2} \mathrm{MnGe}$ (Ref. 11), or $\mathrm{Co}_{2} \mathrm{MnSi}$ (Ref. 12) on $\mathrm{MgO}$-buffered $\mathrm{MgO}(001)$ substrates. Given these epitaxial thin films, we developed fully epitaxial MTJs that had a $\mathrm{Co}_{2} Y Z$ thin film of CCFA, ${ }^{21,26}$ $\mathrm{Co}_{2} \mathrm{MnGe},{ }^{26,27}$ or $\mathrm{Co}_{2} \mathrm{MnSi}$ (Refs. 30-32) as a lower electrode, a $\mathrm{MgO}$ tunnel barrier, and a $\mathrm{Co}_{50} \mathrm{Fe}_{50}$ upper electrode. We reported fully epitaxial CCFA/MgO/ $\mathrm{CO}_{50} \mathrm{Fe}_{50} \mathrm{MTJ}$ showing relatively high TMR ratios of $42 \%$ at RT and $74 \%$ at $55 \mathrm{~K} \cdot{ }^{21,26}$ However, the CCFA thin film composition used in this previous study was $\mathrm{Co}_{2}\left(\mathrm{Cr}_{0.61} \mathrm{Fe}_{0.38}\right) \mathrm{Al}_{0.81}$ and significantly deviated from the stoichiometric one of 2:1:1 (Ref. 26). Hereafter, we refer to the CCFA film with this composition as CCFA film A. We represent the fabricated $\mathrm{Co}_{2} \mathrm{YZ}$ film composition as $\mathrm{Co}_{2} Y_{a} Z_{b}$, where $Y_{a}$ stands for $\mathrm{Cr}_{a 1} \mathrm{Fe}_{a 2}$ $(a=a 1+a 2)$ and $Z_{b}$ stands for $\mathrm{Al}_{b}$ for CCFA; that is, $a$ 
$=0.99, b=0.81$, and $a+b=1.80$ for CCFA film A. Deviation of the film composition from the stoichiometric one inevitably results in structural defects, which may reduce the spin polarization of $E_{F}$ (Refs. 38 and 42). Furthermore, in our previous $\mathrm{CCFA} / \mathrm{MgO} / \mathrm{Co}_{50} \mathrm{Fe}_{50} \mathrm{MTJ}$, the parallel and antiparallel magnetization configurations were controlled by using the difference in the coercive forces between the CCFA lower electrode and the $\mathrm{Co}_{50} \mathrm{Fe}_{50}$ upper electrode (i.e., they were pseudo-spin-valve-type MTJs). This resulted in peaked magnetoresistance versus magnetic field characteristics, ${ }^{21}$ which probably led to TMR ratios lower than they potentially could be. Exchange biasing is favorable for realizing high degrees of the parallel and antiparallel magnetization configurations. $^{43}$

Our purpose in this study was to improve the TMR characteristics of fully epitaxial MTJs with a full-Heusler alloy thin film of CCFA as a lower electrode, a $\mathrm{MgO}$ tunnel barrier, and a $\mathrm{Co}_{50} \mathrm{Fe}_{50}$ upper electrode, and demonstrate a potentially high tunneling spin polarization of CCFA. To do this, we took two approaches. The first was to fabricate pseudo-spin-valve-type, fully epitaxial CCFA/MgO/ $\mathrm{Co}_{50} \mathrm{Fe}_{50}$ MTJs with a CCFA film composition close to the stoichiometric one of $2: 1: 1$. The second was to develop fully epitaxial $\mathrm{CCFA} / \mathrm{MgO} / \mathrm{Co}_{50} \mathrm{Fe}_{50}$ MTJs with exchange biasing.

In Sec. II, we describe our experimental methods. The preparation of MTJ layer structures in an ultrahigh vacuum chamber through the combined use of magnetron sputtering and electron evaporation is described. In Sec. III, we present experimental results regarding structural characterization of fabricated CCFA thin films and CCFA/MgO/ $\mathrm{Co}_{50} \mathrm{Fe}_{50}$ MTJ layer structures. In Sec. III, we also describe the experimentally obtained TMR characteristics of the fully epitaxial CCFA/MgO/ $\mathrm{Co}_{50} \mathrm{Fe}_{50}$ MTJs. The pseudo-spin-valve-type, fully epitaxial MTJs with a CCFA film composition close to the stoichiometric one showed a TMR ratio of $90 \%$ at RT $(240 \%$ at $4.2 \mathrm{~K}){ }^{28}$ We discuss a possible reason for the increased TMR ratio, compared with a previously obtained value of $42 \%$ at RT $(74 \%$ at $55 \mathrm{~K})$ for $\mathrm{CCFA} / \mathrm{MgO} /$ $\mathrm{Co}_{50} \mathrm{Fe}_{50}$ MTJs with a CCFA film composition which significantly deviated from the stoichiometric one, in terms of the increased $B 2$ ratio achieved by bringing the CCFA film composition closer to the stoichiometric one. Furthermore, we have demonstrated a high TMR ratio of $109 \%$ at RT $(317 \%$ at $4.2 \mathrm{~K}$ ) for fully epitaxial $\mathrm{CCFA} / \mathrm{MgO} / \mathrm{Co}_{50} \mathrm{Fe}_{50}$ with exchange biasing. ${ }^{33}$ We have estimated a tunneling spin polarization of 0.88 at $4.2 \mathrm{~K}$ for epitaxial CCFA films with the $B 2$ structure from the experimentally obtained TMR ratios and suggested enhancement of the TMR ratio by a coherent tunneling contribution for fully epitaxial CCFA/MgO/ $\mathrm{Co}_{50} \mathrm{Fe}_{50}$ MTJs. In Sec. IV, we summarize our results and conclude.

\section{EXPERIMENTAL METHODS}

Full-Heusler alloys are ternary intermetallic compounds with the composition $X_{2} Y Z$ in the $L 2_{1}$ cubic structure (space group: $F m \overline{3} m$ ). A typical full-Heusler alloy consists of two different transition metals $X$ and $Y$ and a nonmagnetic element $Z$, as shown in Fig. 1(a). The lattice consists of four
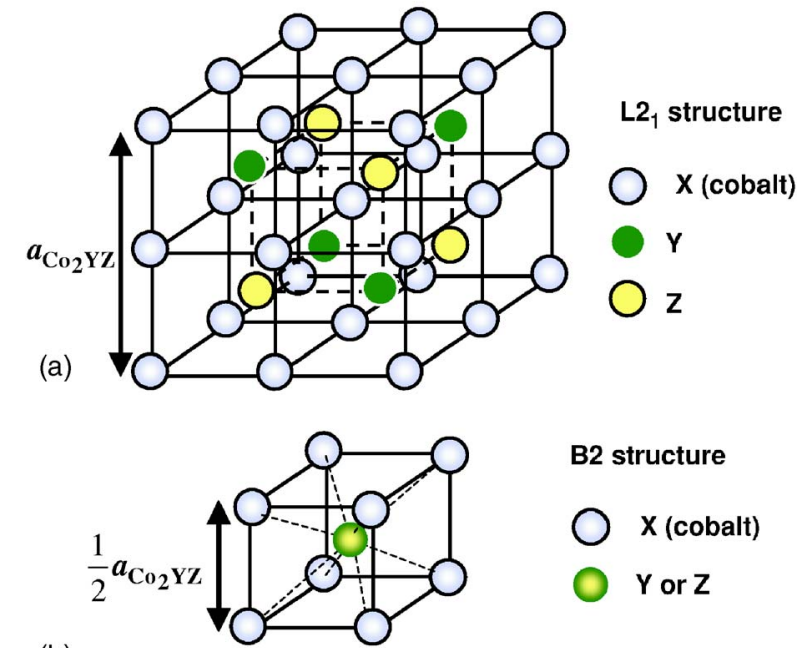

(b)

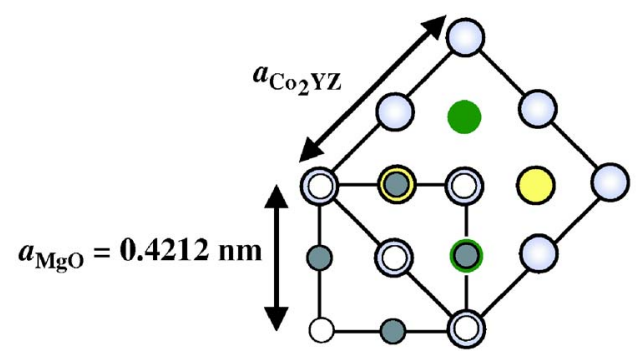

FIG. 1. (Color online) (a) Schematic view of the $L 2_{1}$ crystal structure with composition $X_{2} Y Z$. The lattice consists of four different fcc sublattices. Each has an atom basis as follows: $X$ element at $(0,0,0)$ and $(1 / 2,1 / 2,1 / 2), Y$ at $(1 / 4,1 / 4,1 / 4)$, and $Z$ at $(3 / 4,3 / 4,3 / 4)$. (b) Schematic view of the $B 2$ crystal structure with composition $X_{2} Y Z$. (c) Top view of Heusler alloy crystal structure $\left(L 2_{1}\right.$ structure). $\mathrm{MgO}$ cubic structure is superimposed.

different fcc sublattices. Each has an atom basis as follows: $X$ element at $(0,0,0)$ and $(1 / 2,1 / 2,1 / 2), Y$ at $(1 / 4,1 / 4$, $1 / 4)$, and $Z$ at $(3 / 4,3 / 4,3 / 4)$. The $Y$-Z-type disordered $X_{2} Y Z$ has the $B 2$ structure [Fig. 1(b)]. The lattice mismatch between CCFA [lattice constant $a=0.5737$ nm (Refs. 39 and 40)] and $\mathrm{MgO}(a=0.4212 \mathrm{~nm})$ on a $45^{\circ}$ in-plane rotation is relatively small, about $-3.7 \%$. Therefore, it is reasonable to expect that CCFA films will grow epitaxially with their [100] direction rotated by $45^{\circ}$ from the $\mathrm{MgO}$ [100] direction in the (001) plane [Fig. 1(c)]. Similarly, the lattice mismatch between $\mathrm{Co}_{50} \mathrm{Fe}_{50}$ [lattice constant $a=0.2850 \mathrm{~nm}$ (Ref. 44)] and $\mathrm{MgO}$ on a $45^{\circ}$ in-plane rotation is relatively small, about $-4.3 \%$. We can also expect that $\mathrm{Co}_{50} \mathrm{Fe}_{50}$ films will grow epitaxially on $\mathrm{MgO}$ with the $\mathrm{Co}_{50} \mathrm{Fe}_{50}$ [100] direction rotated by $45^{\circ}$ from the $\mathrm{MgO}$ [100] direction in the (001) plane.

We fabricated two sets of MTJ layer structures. The first set was pseudo-spin-valve-type, fully epitaxial $\mathrm{CCFA} / \mathrm{MgO} / \mathrm{Co}_{50} \mathrm{Fe}_{50}$ MTJs with a CCFA film composition close to the stoichiometric one. For this type of MTJ, we used a wedge-shaped $\mathrm{MgO}$ tunnel barrier so that we could investigate the TMR characteristics as a function of $\mathrm{MgO}$ barrier thickness. The nominal thickness of the $\mathrm{MgO}$ tunnel barrier $\left(t_{\mathrm{MgO}}\right)$ was varied from 1.0 to $3.6 \mathrm{~nm}$ on each 20 $\times 20 \mathrm{~mm}$ substrate by using a linearly moving shutter during the deposition. The fabricated epitaxial MTJ layer structure (from the substrate side) was $\mathrm{MgO}$ buffer layer $(10 \mathrm{~nm}) / \mathrm{CCFA}$ lower electrode $(50 \mathrm{~nm}) / \mathrm{MgO}$ tunnel bar- 
rier $(1.0-3.6 \mathrm{~nm}) / \mathrm{Co}_{50} \mathrm{Fe}_{50}$ upper electrode $(30 \mathrm{~nm})$, grown on a $\mathrm{MgO}(001)$ single-crystal substrate. The $\mathrm{Co}_{50} \mathrm{Fe}_{50}$ layer, which had a coercive force higher than that of the CCFA layer, was deposited at RT using magnetron sputtering. The composition of the CCFA film used in the first set of MTJs was $\mathrm{Co}_{2.0} \mathrm{Cr}_{0.56} \mathrm{Fe}_{0.40} \mathrm{Al}_{0.99}$ as determined through inductively coupled plasma (ICP) analysis with an accuracy of $2 \%-3 \%$ for the composition of each element. We refer to the CCFA film with this composition as CCFA film B $(a=0.96, b$ $=0.99, a+b=1.95)$. Thus, the film composition was brought close to the stoichiometric one of 2:1:1 for $\mathrm{Co}_{2}\left(\mathrm{Cr}_{0.6} \mathrm{Fe}_{0.4}\right) \mathrm{Al}$, in contrast with that of the $\mathrm{Co}_{2} \mathrm{Cr}_{0.61} \mathrm{Fe}_{0.38} \mathrm{Al}_{0.81}$ (CCFA film A, $a+b=1.80)$ used to fabricate the CCFA MTJs which showed TMR ratios of about $42 \%$ at RT. ${ }^{21,26}$

The second set was fully epitaxial CCFA/MgO/ $/ \mathrm{Co}_{50} \mathrm{Fe}_{50}$ MTJs with exchange biasing. We used an upper electrode of $\mathrm{Co}_{50} \mathrm{Fe}_{50}$ film in an antiferromagnetically coupled (i.e., synthetic ferrimagnetic) $\mathrm{Co}_{50} \mathrm{Fe}_{50} / \mathrm{Ru} / \mathrm{Co}_{90} \mathrm{Fe}_{10}$ trilayer exchange-biased by an IrMn antiferromagnetic layer through the $\mathrm{Co}_{90} \mathrm{Fe}_{10} / \mathrm{IrMn}$ interface to obtain a high value of exchange-bias field $\left(H_{\mathrm{ex}}\right)$ for epitaxial $\mathrm{Co}_{50} \mathrm{Fe}_{50}$ electrodes. ${ }^{45}$ We confirmed that the $\mathrm{Co}_{50} \mathrm{Fe}_{50} / \mathrm{Ru} / \mathrm{Co}_{90} \mathrm{Fe}_{10}$ trilayers, which had a typical $\mathrm{Ru}$ interlayer thickness of $0.8 \mathrm{~nm}$, behaved magnetically as synthetic ferrimagnets. The fabricated MTJ layer structure in this case was as follows: (from the substrate side) $\mathrm{MgO}$ buffer layer $(10 \mathrm{~nm}) / \mathrm{CCFA} \quad(50 \mathrm{~nm}) / \mathrm{MgO}$ barrier $(2.4 \mathrm{~nm}) / \mathrm{Co}_{50} \mathrm{Fe}_{50} \quad(3.4 \mathrm{~nm}) / \mathrm{Ru} \quad(0.8 \mathrm{~nm}) / \mathrm{Co}_{90} \mathrm{Fe}_{10}$ $(2 \mathrm{~nm}) / \mathrm{IrMn}(10 \mathrm{~nm}) / \mathrm{Ru}$ cap $(5 \mathrm{~nm})$. The 5 -nm-thick Ru cap layer was used to protect the IrMn layer from corrosion. The composition of the CCFA film used in the second set of MTJs was determined through ICP analysis to be $\mathrm{Co}_{2} \mathrm{Cr}_{0.57} \mathrm{Fe}_{0.39} \mathrm{Al}_{1.12}$. We refer to the CCFA film with this composition as CCFA film C $(a=0.96, b=1.12, a+b=2.08)$.

All layers in these MTJs were successively deposited on $\mathrm{MgO}(001)$ single-crystal substrates in an ultrahigh vacuum chamber (with a base pressure of about $8 \times 10^{-8} \mathrm{~Pa}$ ) through the combined use of magnetron sputtering and electron beam evaporation. The CCFA lower electrode was deposited by $\mathrm{rf}$ magnetron sputtering at RT and subsequently annealed in situ at $500{ }^{\circ} \mathrm{C}$ for $15 \mathrm{~min}$. The $\mathrm{MgO}$ tunnel barrier was deposited by electron beam evaporation at RT. The pressure during the deposition of the $\mathrm{MgO}$ tunnel barrier was about $6 \times 10^{-7} \mathrm{~Pa}$. The layers of $\mathrm{Co}_{50} \mathrm{Fe}_{50}, \mathrm{Ru}, \mathrm{Co}_{90} \mathrm{Fe}_{10}$, and IrMn were all deposited by magnetron sputtering at RT.

The structural characterization for the fabricated CCFA thin films and CCFA MTJs were performed using in situ reflection high-energy electron diffraction (RHEED), x-ray Bragg scans, $\mathrm{x}$-ray pole figure measurements (Bruker AXS D8 DISCOVER Hybrid), cross-sectional high-resolution transmission electron microscope (HRTEM) observation, and transmission electron diffraction.

We fabricated MTJs with the fully epitaxial layer structures described above by photolithography and Ar ion milling. The fabricated junction sizes were $8 \times 8 \mu \mathrm{m}^{2}$ for the first set of MTJs and $10 \times 10 \mu \mathrm{m}^{2}$ for the second set. After the microfabrication procedure, some of the MTJs were annealed at $175^{\circ} \mathrm{C}$ for $1 \mathrm{~h}$ in a vacuum of $10^{-4} \mathrm{~Pa}$ under a magnetic field of $5 \mathrm{kOe}$. The magnetoresistance was mea-

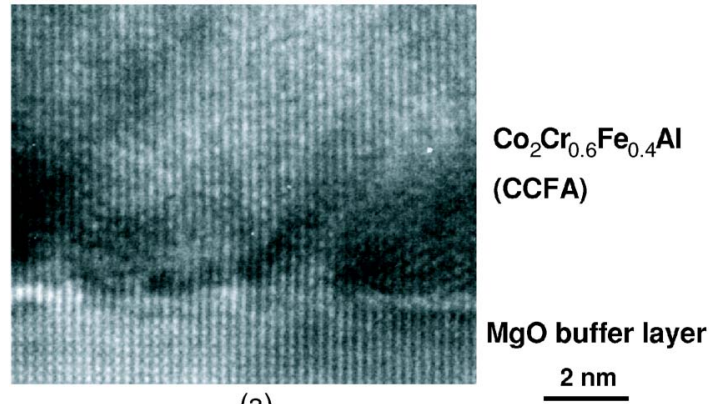

(a)

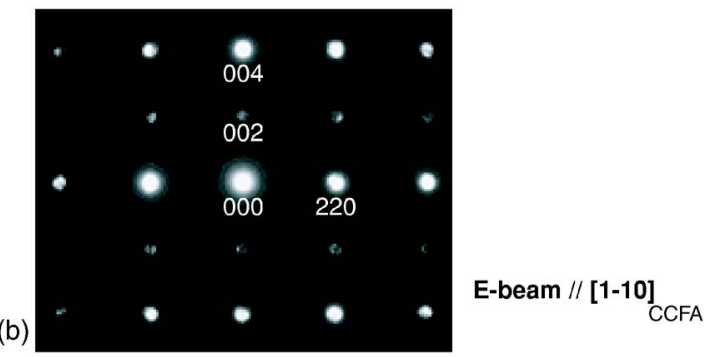

FIG. 2. (Color online) (a) Cross-sectional high-resolution transmission electron microscope image of $\mathrm{Co}_{2} \mathrm{Cr}_{0.6} \mathrm{Fe}_{0.4} \mathrm{Al}$ (CCFA) $(50 \mathrm{~nm}) / \mathrm{MgO}$ buffer layer, along the [1-10] direction of the CCFA. (b) Electron diffraction pattern for the CCFA layer. The electron-beam diameter was $10 \mathrm{~nm}$.

sured with a magnetic field applied along the [110] axis of the CCFA at temperatures from $4.2 \mathrm{~K}$ to RT using a dc fourprobe method. We defined the TMR ratio as $\left(R A_{\mathrm{AP}}\right.$ $\left.-R A_{\mathrm{P}}\right) / R A_{\mathrm{P}}$, where $R A_{\mathrm{AP}}$ and $R A_{\mathrm{P}}$ are the respective resistance-area products for the antiparallel and parallel magnetization configurations between the upper and lower electrodes.

\section{EXPERIMENTAL RESULTS AND DISCUSSION}

\section{A. Structural properties of CCFA thin films and $\mathrm{CCFA} / \mathrm{MgO} / \mathrm{Co}_{50} \mathrm{Fe}_{50} \mathrm{MTJ}$ layer structures}

We will first describe the structural properties of the fabricated CCFA thin films. We used $\mathrm{x}$-ray pole figure measurements to confirm that the fabricated 50-nm-thick CCFA thin films with CCFA films B and $\mathrm{C}$ annealed in situ at $500{ }^{\circ} \mathrm{C}$ were grown epitaxially on $\mathrm{MgO}$-buffered $\mathrm{MgO}(001)$ substrates and crystallized in the $B 2$ structure. The crystallographic relationship was CCFA (001) [100]\|MgO (001) [011]. These results were in agreement with our previous work. ${ }^{21,26}$ Figure 2(a) shows a cross-sectional high-resolution TEM lattice image, along the [1-10] direction of the CCFA film, of a $500{ }^{\circ} \mathrm{C}$ annealed CCFA $(50 \mathrm{~nm})$ thin film (CCFA film B) deposited on a $\mathrm{MgO}$ buffer layer. As can be seen in Fig. 2(a), the TEM lattice image clearly showed that the CCFA film was grown epitaxially on a MgO-buffered $\mathrm{MgO}(001)$ substrate and was single crystalline. Figure 2(b) shows a microbeam electron diffraction pattern with a beam diameter of $10 \mathrm{~nm}$ for the CCFA film. In the electron diffraction pattern, 002 spots were observed, while 111 spots were not observed. These results-i.e., the existence of 002 spots and the lack of 111 spots-indicated that the $500{ }^{\circ} \mathrm{C}$ annealed CCFA films with the film composition of $\mathrm{Co}_{2.0} \mathrm{Cr}_{0.56} \mathrm{Fe}_{0.40} \mathrm{Al}_{0.99}$ grown on $\mathrm{MgO}$ buffer layers had the 

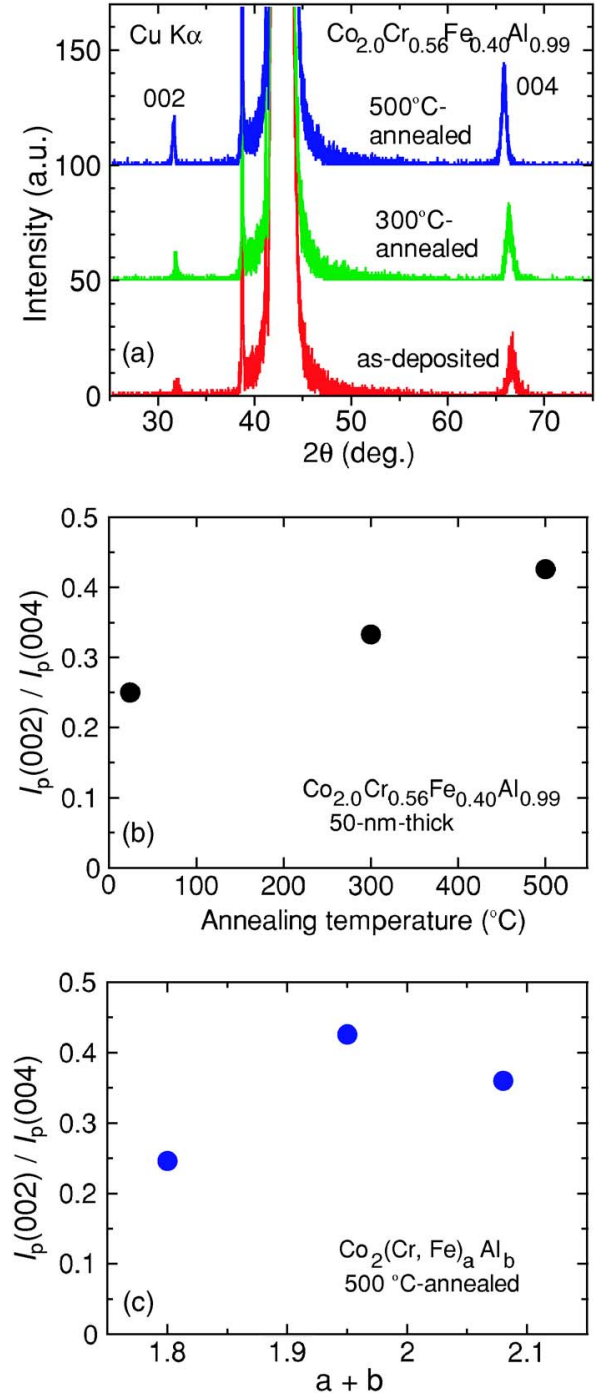

FIG. 3. (Color online) (a) X-ray $\theta-2 \theta$ diffraction patterns of 50-nm-thick as-deposited, $300{ }^{\circ} \mathrm{C}$ annealed, and $500{ }^{\circ} \mathrm{C}$ annealed CCFA films deposited on $\mathrm{MgO}$-buffered $\mathrm{MgO}(001)$ substrates. The film composition was $\mathrm{Co}_{2.0} \mathrm{Cr}_{0.56} \mathrm{Fe}_{0.40} \mathrm{Al}_{0.99}$. (b) Peak intensity ratio of $I_{\mathrm{P}}(002) / I_{\mathrm{P}}(004)$ for the CCFA thin films [these are the same films shown in (a)] as a function of in situ postdeposition annealing temperature. (c) Peak intensity ratio of $I_{\mathrm{P}}(002) / I_{\mathrm{P}}(004)$ for the CCFA thin films annealed in situ at $500^{\circ} \mathrm{C}$ as a function of the film composition $a+b$ in the representation composition of $\mathrm{Co}_{2}(\mathrm{Cr}, \mathrm{Fe})_{a} \mathrm{Al}_{b}$.

$B 2$ structure, and so were consistent with the results obtained through $\mathrm{x}$-ray pole figure measurements.

Figure 3(a) shows x-ray $\theta-2 \theta$ diffraction patterns of 50-nm-thick as-deposited, $300{ }^{\circ} \mathrm{C}$ and $500{ }^{\circ} \mathrm{C}$ annealed CCFA films [film composition: $\mathrm{Co}_{2.0} \mathrm{Cr}_{0.56} \mathrm{Fe}_{0.40} \mathrm{Al}_{0.99}$ (CCFA film B)]; clear 002 and 004 peaks were observed even for the as-deposited films. As shown in Fig. 3(a), annealing the asdeposited CCFA film at temperatures ranging from 300 to $500{ }^{\circ} \mathrm{C}$ caused the intensities $\left(I_{\mathrm{P}}\right)$ of 002 and 004 peaks to increase. This result was similar to that for the $\mathrm{Co}_{2} \mathrm{Cr}_{0.61} \mathrm{Fe}_{0.38} \mathrm{Al}_{0.81}$ (CCFA film A) used to fabricate CCFA MTJs which showed TMR ratios of about $42 \%$ at RT. ${ }^{21,26}$ Furthermore, the peak intensity ratio of $I_{\mathrm{P}}(002) / I_{\mathrm{P}}(004)$ rose with increasing annealing temperature [Fig. 3(b)]. This suggests that the ratio of the $B 2$ structure region to the $A 2$ structure region was increased by in situ postdeposition annealing.
We next investigated the relative intensities of $x$-ray diffraction 002 and 004 peaks for CCFA films as a function of the film composition. Note that the 002 peak is specific to the $B 2$ structure. If we use a parameter $q$ to represent the ratio of the 002 peak intensity, $I_{\mathrm{P}}(002)$, to the 004 peak intensity, $I_{\mathrm{P}}(004), q$ corresponds to the ratio of the $B 2$ region to the $A 2$ region (the $B 2$ ratio).

$$
q=\frac{I_{\mathrm{P}}(002)}{I_{\mathrm{P}}(004)} .
$$

Figure 3(c) shows the peak intensity ratio of $I_{\mathrm{P}}(002) / I_{\mathrm{P}}(004)$ for the CCFA films $(50 \mathrm{~nm})$ deposited at RT on $\mathrm{MgO}$ buffer layers $(10 \mathrm{~nm})$ and subsequently annealed in situ at $500{ }^{\circ} \mathrm{C}$ as a function of the film composition of $a$ $+b$. Compared to the peak intensity ratio of $I_{\mathrm{P}}(002) / I_{\mathrm{P}}(004)$ for our previous CCFA thin film with a film composition which deviated significantly from the stoichiometric one of 2:1:1 (CCFA film A: $\mathrm{Co}_{2} \mathrm{Cr}_{0.61} \mathrm{Fe}_{0.38} \mathrm{Al}_{0.81}, a+b=1.80$ ), that of the CCFA thin film with a composition close to the stoichiometric one (CCFA film $\mathrm{B}, \mathrm{Co}_{2.0} \mathrm{Cr}_{0.56} \mathrm{Fe}_{0.40} \mathrm{Al}_{0.99}, a$ $+b=1.95)$ was markedly high. As shown in Fig. 3(c), the parameter $q$ corresponding to the $B 2$ ratio increased as the film composition approached the stoichiometric one of 2:1:1.

We will now describe the structural properties of the fabricated MTJ structures. First, we describe the structural properties of the fabricated pseudo-spin-valve-type, fully epitaxial CCFA/MgO/ $\mathrm{Co}_{50} \mathrm{Fe}_{50}$ MTJs with a CCFA film composition close to the stoichiometric one (CCFA film B). RHEED patterns observed in situ for each layer during fabrication clearly indicated that the CCFA lower electrode, $\mathrm{MgO}$ tunnel barrier, and $\mathrm{Co}_{50} \mathrm{Fe}_{50}$ upper electrode grew epitaxially. We also performed a cross-sectional high-resolution TEM observation for a CCFA (50 nm)/MgO $(2 \mathrm{~nm}) / \mathrm{Co}_{50} \mathrm{Fe}_{50}(30 \mathrm{~nm})$ MTJ layer structure along the [110] direction of the CCFA film. The observed TEM lattice image clearly showed that all the layers of the $\mathrm{CCFA} / \mathrm{MgO} / \mathrm{Co}_{50} \mathrm{Fe}_{50}$ MTJ structure were grown epitaxially and were single crystalline. It also confirmed that extremely smooth and abrupt interfaces were formed. ${ }^{28}$

Second, we will describe the structural properties of the fully epitaxial $\mathrm{CCFA} / \mathrm{MgO} / \mathrm{Co}_{50} \mathrm{Fe}_{50}$ MTJs with exchange biasing. In the MTJ structure, CCFA films with the composition of $\mathrm{Co}_{2} \mathrm{Cr}_{0.57} \mathrm{Fe}_{0.39} \mathrm{Al}_{1.12}$ (CCFA film $\mathrm{C}$ ) were used. The basic tunnel junction trilayers of the CCFA/ $\mathrm{MgO} / \mathrm{Co}_{50} \mathrm{Fe}_{50}$ MTJs with exchange biasing were the same as those of the pseudo-spin-valve-type MTJs described above, except that the thickness of the $\mathrm{Co}_{50} \mathrm{Fe}_{50}$ upper electrode $\left(t_{\mathrm{CoFe}}\right)$ was decreased to $3.4 \mathrm{~nm}$ from $t_{\mathrm{CoFe}}$ of $30 \mathrm{~nm}$. RHEED patterns observed in situ for each layer during fabrication clearly indicated that the CCFA lower electrode, $\mathrm{MgO}$ tunnel barrier, and $\mathrm{Co}_{50} \mathrm{Fe}_{50}$ upper electrode grew epitaxially. Figure 4 shows a cross-sectional high-resolution TEM lattice image of the fabricated MTJ layer structure from the CCFA layer to the IrMn layer. This cross-sectional HRTEM image clearly shows that all the layers of the CCFA/ $\mathrm{MgO} / \mathrm{Co}_{50} \mathrm{Fe}_{50}$ basic tunnel junction trilayer were grown epitaxially and were single crystalline. It also confirmed that extremely smooth and abrupt interfaces were formed. 


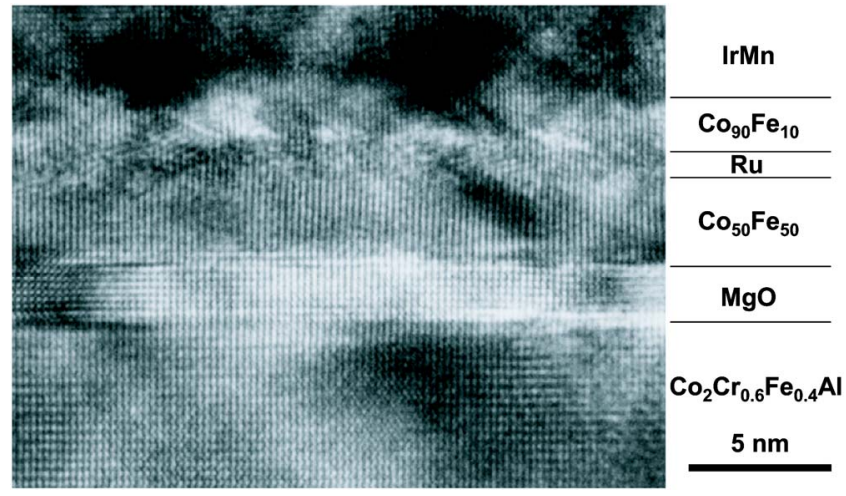

FIG. 4. (Color online) Cross-sectional high-resolution transmission electron microscope image of a MTJ layer structure, consisting of $\mathrm{Co}_{2} \mathrm{Cr}_{0.6} \mathrm{Fe}_{0.4} \mathrm{Al}$ (CCFA) $(50 \mathrm{~nm}) / \mathrm{MgO}(2 \mathrm{~nm}) / \mathrm{Co}_{50} \mathrm{Fe}_{50}(3 \mathrm{~nm}) / \mathrm{Ru}(0.8 \mathrm{~nm}) / \mathrm{Co}_{90} \mathrm{Fe}_{10}$ $(2 \mathrm{~nm}) / \mathrm{IrMn}(10 \mathrm{~nm}) / \mathrm{Ru}$ cap $(5 \mathrm{~nm})$, along the [1-10] direction of the CCFA.

Next, we will describe the structural properties of the $\mathrm{Co}_{50} \mathrm{Fe}_{50} / \mathrm{Ru} / \mathrm{Co}_{90} \mathrm{Fe}_{10} / \mathrm{IrMn}$ quadrilayers. We also observed streak patterns in the RHEED patterns, dependent on the incident directions of the electron beam, for layers of $\mathrm{Ru}$, $\mathrm{Co}_{90} \mathrm{Fe}_{10}$, and IrMn, indicating that the layers grew epitaxially on the epitaxial $\mathrm{Co}_{50} \mathrm{Fe}_{50}$ electrode. Furthermore, crosssectional HRTEM lattice images (Fig. 4) clearly showed that all the layers of $\mathrm{Ru}, \mathrm{Co}_{90} \mathrm{Fe}_{10}$, and $\mathrm{IrMn}$ were also grown epitaxially on the single-crystal $\mathrm{Co}_{50} \mathrm{Fe}_{50}$ electrode and were single crystalline, although the detailed structures of the successive layers from the $\mathrm{Ru}$ interlayer to the IrMn layer were not fully analyzed.

\section{B. TMR characteristics of pseudo-spin-valve-type CCFA/MgO/ $/ \mathrm{Co}_{50} \mathrm{Fe}_{50}$ MTJs with a CCFA film composition close to the stoichiometric one}

We will describe the TMR characteristics of the fabricated pseudo-spin-valve-type, fully epitaxial $\mathrm{CCFA} / \mathrm{MgO} /$ $\mathrm{Co}_{50} \mathrm{Fe}_{50}$ MTJs with a CCFA film composition of $\mathrm{Co}_{2.0} \mathrm{Cr}_{0.56} \mathrm{Fe}_{0.40} \mathrm{Al}_{0.99}$ (CCFA thin film B), which was close to the stoichiometric one. The as-fabricated (i.e., not ex situ annealed) MTJs exhibited typical TMR ratios of $80 \%$ at RT and $210 \%$ at $4.2 \mathrm{~K}$. Figure 5 shows $R A_{\mathrm{P}}$ and TMR ratios of the as-fabricated MTJs at RT as a function of $t_{\mathrm{MgO}}$. A clear exponential dependence of $R A_{\mathrm{P}}$ on $t_{\mathrm{MgO}}$ was observed for a $t_{\mathrm{MgO}}$ range from 1.1 to $2.5 \mathrm{~nm}$, indicating typical tunnel junction behavior. The $m^{*} \varphi_{0}$ value estimated from the slope of $\ln \left(R A_{\mathrm{P}}\right)$ vs $t_{\mathrm{MgO}}$ according to the Wenzel-Kramer-Brillouin (WKB) approximation was $0.32 \mathrm{eV}$ for the CCFA MTJ, where $m^{*}$ is the effective electron mass normalized by the bare electron mass and $\varphi_{0}$ is the potential barrier height (the energy difference between the Fermi level of the emitter ferromagnetic electrode and the bottom of the conduction band in the tunnel barrier). ${ }^{46}$ This value was close to that of $0.39 \mathrm{eV}$ obtained for epitaxial $\mathrm{Fe} / \mathrm{MgO} / \mathrm{Fe} \mathrm{MTJs}^{47}{ }^{4}$ Relatively high TMR ratios from $60 \%$ to $83 \%$ were obtained at RT for a wide range of $t_{\mathrm{MgO}}$ from 1.1 to $2.5 \mathrm{~nm}$.

The as-fabricated (i.e., not ex situ annealed) MTJs exhibited typical TMR ratios of $80 \%$ at RT and $210 \%$ at $4.2 \mathrm{~K}$. Figure 6 shows typical magnetoresistance curves at $V$ $=5 \mathrm{mV}$ at $\mathrm{RT}$ and $4.2 \mathrm{~K}$ for a MTJ postfabrication annealed

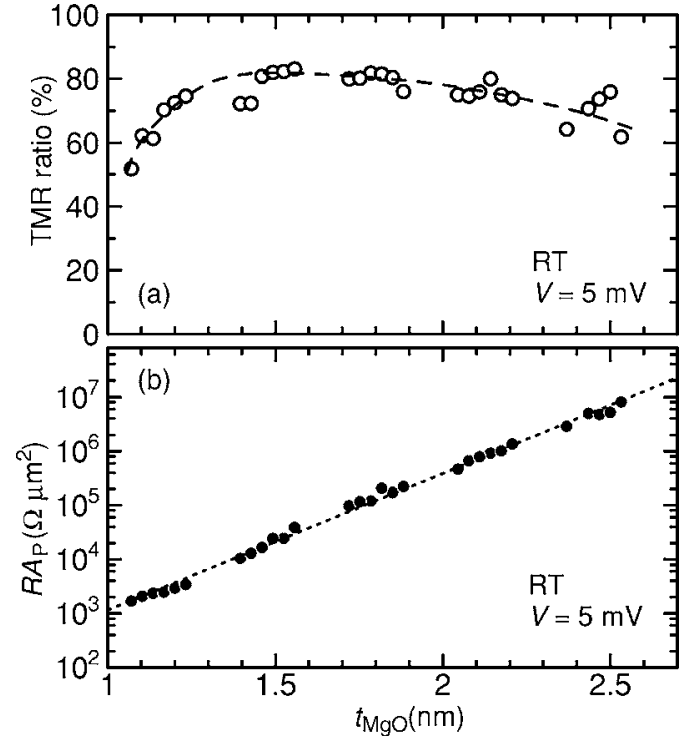

FIG. 5. MgO tunnel barrier thickness $\left(t_{\mathrm{MgO}}\right)$ dependence of (a) TMR ratio and (b) $R A_{\mathrm{P}}$ at RT (measured at a bias voltage of $5 \mathrm{mV}$ ) for as-fabricated (i.e., not ex situ annealed) $\mathrm{Co}_{2} \mathrm{Cr}_{0.6} \mathrm{Fe}_{0.4} \mathrm{Al} / \mathrm{MgO} / \mathrm{Co}_{50} \mathrm{Fe}_{50}$ MTJs. $R A_{\mathrm{P}}$ represents the resistance-area product for the parallel magnetization configuration. The scale of the vertical axis for $R A_{\mathrm{P}}$ is logarithmic. The dashed line serves as a guide to the eye. The dotted line represents a least-squares approximation of the form $\ln \left(R A_{\mathrm{P}}\right)=A+B \cdot t_{\mathrm{MgO}}$.

at $175^{\circ} \mathrm{C}$. The $\mathrm{MgO}$ tunnel barrier thickness was $1.6 \mathrm{~nm}$. The annealed MTJs showed increased TMR ratios of $90 \%$ at RT and $240 \%$ at $4.2 \mathrm{~K}$. The significant increase of the TMR ratio in the epitaxial CCFA MTJs compared to our previously reported value of about $42 \%$ at RT (Refs. 21 and 26) indicates that a film composition close to the stoichiometric one is essential to obtain high spin polarizations in CCFA thin films.

Now we will discuss a possible reason for the improved TMR ratios of the MTJs with a CCFA film having a composition close to the stoichiometric one. As described in Sec. III A, our x-ray diffraction (XRD) analysis for the epitaxially

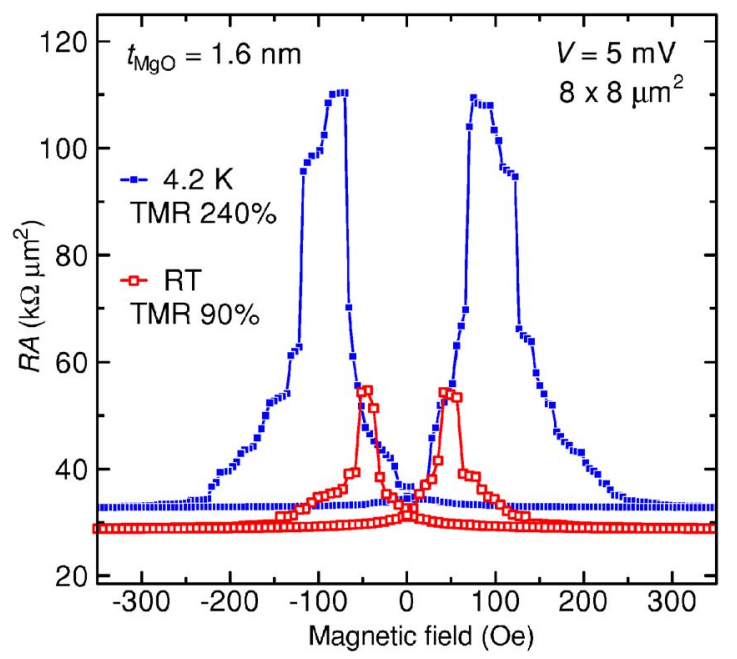

FIG. 6. (Color online) Typical magnetoresistance curves for a pseudo-spinvalve-type, epitaxial $\mathrm{Co}_{2} \mathrm{Cr}_{0.6} \mathrm{Fe}_{0.4} \mathrm{Al} / \mathrm{MgO} / \mathrm{Co}_{50} \mathrm{Fe}_{50}$ MTJ ex situ annealed at $175^{\circ} \mathrm{C}\left(t_{\mathrm{MgO}}=1.6 \mathrm{~nm}\right)$ at a bias voltage of $5 \mathrm{mV}$ at $4.2 \mathrm{~K}$ and RT. The junction size was $8 \times 8 \mu \mathrm{m}^{2}$. The TMR ratios were $90 \%(\mathrm{RT})$ and $240 \%$ (4.2 K). 


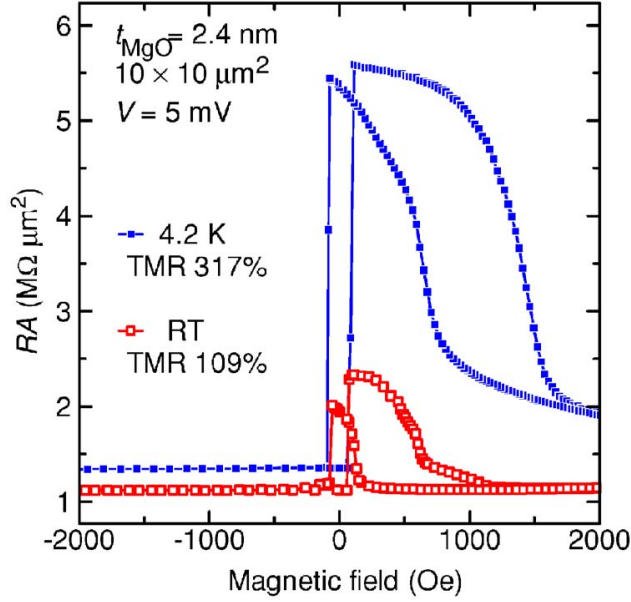

FIG. 7. (Color online) Typical magnetoresistance curves at RT and $4.2 \mathrm{~K}$ for a fully epitaxial $\mathrm{Co}_{2} \mathrm{Cr}_{0.6} \mathrm{Fe}_{0.4} \mathrm{Al} / \mathrm{MgO}(2.4 \mathrm{~nm}) / \mathrm{Co}_{50} \mathrm{Fe}_{50}$ MTJ with exchange biasing.

grown CCFA films indicated that the ratio of the $B 2$ structure region to the $A 2$ structure region (the $B 2$ ratio) was increased by bringing the CCFA film composition close to the stoichiometric one of $a+b=2.0$. Theoretically, it was predicted that A2-type disorder would significantly decrease the spin polarization in $\mathrm{Co}_{2} \mathrm{CrAl}$ while $\mathrm{B} 2$-type disorder would have little effect. $^{38}$ Therefore, we can reasonably attribute the observed TMR improvement to the increased spin polarization at $E_{F}$ due to the increased $B 2$ ratio.

\section{TMR characteristics of CCFA/MgO/ $\mathrm{Co}_{50} \mathrm{Fe}_{50} \mathrm{MTJs}$ with exchange biasing}

We will now describe the TMR characteristics of the fabricated fully epitaxial $\mathrm{CCFA} / \mathrm{MgO} / \mathrm{Co}_{50} \mathrm{Fe}_{50}$ MTJs with exchange biasing. In the MTJ structure, CCFA films with the composition of $\mathrm{Co}_{2} \mathrm{Cr}_{0.57} \mathrm{Fe}_{0.39} \mathrm{Al}_{1.12}$ (CCFA film $\mathrm{C}, a+b$ $=2.08$ ) were used.

Figure 7 shows typical magnetoresistance curves at RT and $4.2 \mathrm{~K}$ of a fabricated fully epitaxial, exchange-biased $\mathrm{CCFA} / \mathrm{MgO} / \mathrm{Co}_{50} \mathrm{Fe}_{50} \mathrm{MTJ}$. The applied bias voltage was $5 \mathrm{mV}$. The MTJ exhibited clear exchange-biased TMR characteristics with high TMR ratios of $109 \%$ at RT and $317 \%$ at $4.2 \mathrm{~K}$. These values are significantly higher than the values of $90 \%$ at RT and $240 \%$ at $4.2 \mathrm{~K}$ for the pseudo-spin-valvetype, fully epitaxial $\mathrm{CCFA} / \mathrm{MgO} / \mathrm{Co}_{50} \mathrm{Fe}_{50}$ MTJs described in Sec. III B. We obtained relatively high $H_{\mathrm{ex}}$ values of about $350 \mathrm{Oe}$ at RT and about $1000 \mathrm{Oe}$ at $4.2 \mathrm{~K}$, as shown in Fig. 7. We can reasonably attribute the high $H_{\mathrm{ex}}$ values obtained for the fabricated MTJs to a lower net saturation magnetization of the synthetic ferrimagnetic trilayer compared with a saturation magnetization of the $\mathrm{Co}_{50} \mathrm{Fe}_{50}$ electrode.

Figure 8 shows the TMR ratio for a fabricated fully epitaxial $\mathrm{CCFA} / \mathrm{MgO} / \mathrm{Co}_{50} \mathrm{Fe}_{50} \mathrm{MTJ}$ (CCFA MTJ) with exchange biasing as a function of temperature $(T)$ from $4.2 \mathrm{~K}$ to RT (the same MTJ shown in Fig. 7). For comparison, the TMR ratio as a function of $T$ is also plotted for a fully epitaxial $\mathrm{Co}_{50} \mathrm{Fe}_{50} / \mathrm{MgO} / \mathrm{Co}_{50} \mathrm{Fe}_{50}$ MTJ with exchange biasing (reference $\mathrm{Co}_{50} \mathrm{Fe}_{50} \mathrm{MTJ}$ ) identically fabricated with the same layer structure as that of the exchange-biased CCFA

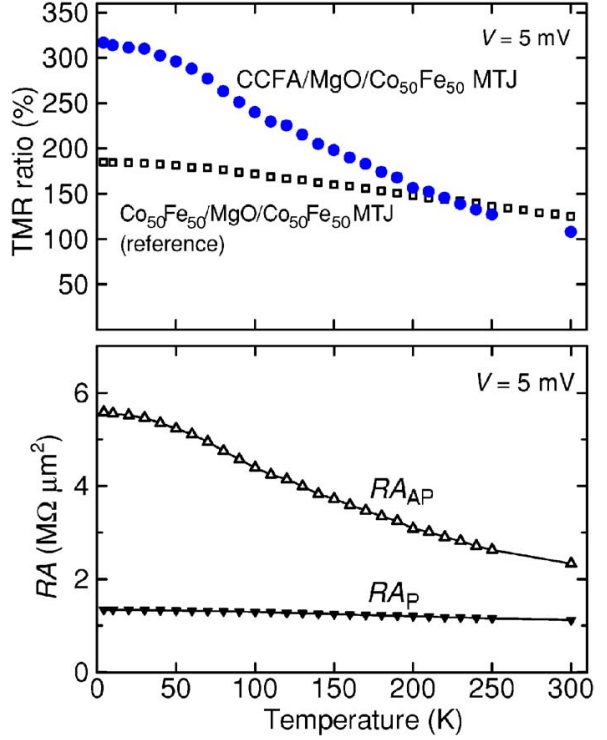

FIG. 8. (Color online) TMR ratios and $R A_{\mathrm{AP}}$ and $R A_{\mathrm{P}}$ for a fully epitaxial, exchange-biased $\mathrm{Co}_{2} \mathrm{Cr}_{0.6} \mathrm{Fe}_{0.4} \mathrm{Al} / \mathrm{MgO} / \mathrm{Co}_{50} \mathrm{Fe}_{50} \mathrm{MTJ}$ as a function of temperature from $4.2 \mathrm{~K}$ to $\mathrm{RT}$, where $R A_{\mathrm{AP}}$ and $R A_{\mathrm{P}}$ represent the respective junction resistance-area products for the antiparallel and parallel magnetization configurations.

MTJ except that the lower electrode CCFA was replaced with $\mathrm{Co}_{50} \mathrm{Fe}_{50}$. The $\mathrm{Co}_{50} \mathrm{Fe}_{50}$ MTJs were postfabrication annealed under the same annealing conditions as for the CCFA MTJs (i.e., at $175^{\circ} \mathrm{C}$ under a magnetic field of $5 \mathrm{kOe}$ ). The layer structure (from the substrate side) was $\mathrm{MgO}$ buffer layer $(10 \mathrm{~nm}) / \mathrm{Co}_{50} \mathrm{Fe}_{50}(50 \mathrm{~nm}) / \mathrm{MgO}(2.2 \mathrm{~nm}) / \mathrm{Co}_{50} \mathrm{Fe}_{50}$ $(3 \mathrm{~nm}) / \mathrm{Ru} \quad(0.8 \mathrm{~nm}) / \mathrm{Co}_{90} \mathrm{Fe}_{10} \quad(2 \mathrm{~nm}) / \mathrm{IrMn} \quad(10 \mathrm{~nm}) / \mathrm{Ru}$ $(5 \mathrm{~nm})$, grown on a $\mathrm{MgO}(001)$ single-crystal substrate. The $\mathrm{Co}_{50} \mathrm{Fe}_{50}$ MTJs showed TMR ratios of $185 \%$ at $4.2 \mathrm{~K}$ and $125 \%$ at RT. As shown in Fig. 8, the TMR ratio of the CCFA MTJ was higher than that of the $\mathrm{Co}_{50} \mathrm{Fe}_{50}$ MTJ below about $220 \mathrm{~K}$, although it was slightly lower at RT. The comparison of the $T$ dependences of the TMR ratios of the CCFA MTJ and $\mathrm{CO}_{50} \mathrm{Fe}_{50}$ MTJ definitely indicated that the higher TMR ratio for the CCFA MTJ below about $200 \mathrm{~K}$, which reached $317 \%$ at $4.2 \mathrm{~K}$, was due to a high tunneling spin polarization of the epitaxial CCFA electrode.

We next estimated the tunneling spin polarization of the epitaxial CCFA electrode from the obtained TMR ratios. The TMR ratios for MTJs have been traditionally related to the spin polarizations at $E_{F}, P_{1}$, and $P_{2}$, of the ferromagnetic electrodes through Jullière's model; ${ }^{48}$ i.e.,

$$
\mathrm{TMR}=2 P_{1} P_{2} /\left(1-P_{1} P_{2}\right) .
$$

Jullière's model was derived by assuming a loss of coherence in tunneling (i.e., nonconservation of the electron's wave vector component parallel to the interface). ${ }^{49}$ However, a straightforward application of Jullière's model for a TMR ratio of $317 \%$ at $4.2 \mathrm{~K}$ for the fully epitaxial CCFA $/ \mathrm{MgO} / \mathrm{Co}_{50} \mathrm{Fe}_{50}$ MTJ with a $\mathrm{Co}_{50} \mathrm{Fe}_{50}$ electrode spin polarization of 0.50 , derived from $d I / d V$ curves of superconductor $/ \mathrm{AlO}_{x} / \mathrm{Co}_{50} \mathrm{Fe}_{50}$ tunnel structures, ${ }^{50}$ corresponding to the originally defined spin polarization using majority- and minority-spin band density of states at $E_{F}$, results in an unrealistically high $P$ value exceeding 1.0 for the 
epitaxial CCFA electrode. This result indicates enhancement of the TMR ratio by a coherent tunneling contribution ${ }^{51,52}$ for fully epitaxial CCFA/MgO/ $/ \mathrm{Co}_{50} \mathrm{Fe}_{50}$ MTJs. Furthermore, the obtained TMR ratios of $185 \%$ at $4.2 \mathrm{~K}$ and $125 \%$ at RT clearly indicate enhancement of the TMR ratio by a coherent tunneling contribution for the reference $\mathrm{Co}_{50} \mathrm{Fe}_{50}$ MTJs. Therefore, we estimated the tunneling spin polarization, or effective spin polarization for the epitaxial $\mathrm{CO}_{50} \mathrm{Fe}_{50}$ electrodes, $P_{\mathrm{CoFe}}$, by applying Jullière's model for the TMR ratio of $185 \%$ at $4.2 \mathrm{~K}\left(125 \%\right.$ at RT) of the reference $\mathrm{Co}_{50} \mathrm{Fe}_{50}$ MTJ. We obtained a $P_{\mathrm{CoFe}}$ value of 0.69 at $4.2 \mathrm{~K}(0.62$ at RT), which was higher than the above $P$ value of 0.50 derived from superconductor/ $\mathrm{AlO}_{x} / \mathrm{Co}_{50} \mathrm{Fe}_{50}$ tunnel structures. ${ }^{50}$ Similarly, we estimated the tunneling spin polarization, or effective spin polarization for an epitaxial CCFA electrode in fully epitaxial $\mathrm{CCFA} / \mathrm{MgO} / \mathrm{Co}_{50} \mathrm{Fe}_{50}$ MTJs, $P_{\text {CCFA }}$, by applying Jullière's model for the TMR ratio of $317 \%$ at $4.2 \mathrm{~K}(109 \%$ at RT) of the CCFA MTJ, along with a $P_{\mathrm{CoFe}}$ value of 0.69 at $4.2 \mathrm{~K}(0.62$ at RT) derived from the TMR ratio for the reference $\mathrm{Co}_{50} \mathrm{Fe}_{50} \mathrm{MTJ}$; in this case, we obtained a high tunneling spin polarization of 0.88 at $4.2 \mathrm{~K}$ ( 0.57 at RT) for the epitaxial CCFA thin film with the $B 2$ structure. Although a rigorous comparison is not justified, the thus obtained $P_{\mathrm{CCFA}}$ value of 0.88 is larger than the theoretically predicted $P_{\text {CCFA }}$ value of 0.78 (Ref. 38) even though we assumed an effective spin polarization of 0.69 at $4.2 \mathrm{~K}(0.62$ at RT) for the epitaxial $\mathrm{Co}_{50} \mathrm{Fe}_{50}$ electrode in the estimation rather than 0.50 at $4.2 \mathrm{~K}$ which was derived from superconductor/ $/ \mathrm{AlO}_{x} / \mathrm{Co}_{50} \mathrm{Fe}_{50}$ tunnel structures. This result also indicates a coherent tunneling contribution ${ }^{51,52}$ for fully epitaxial CCFA $/ \mathrm{MgO} / \mathrm{Co}_{50} \mathrm{Fe}_{50}$ MTJs.

Last, we will discuss the $T$ dependence of the TMR ratio of the fully epitaxial CCFA/MgO/ $\mathrm{Co}_{50} \mathrm{Fe}_{50}$ MTJs with exchange biasing. If we use parameter $\gamma=\alpha(4.2 \mathrm{~K}) / \alpha(\mathrm{RT})$, where $\alpha$ is the TMR ratio, to represent the degree of the TMR ratio's $T$ dependence, $\gamma$ for the CCFA MTJs was 2.9. This $\gamma$ value was higher than the value of 2.1 previously reported for $\mathrm{CMS} / \mathrm{MgO} / \mathrm{Co}_{50} \mathrm{Fe}_{50}$ MTJs $[\alpha(\mathrm{RT})=90 \%$ and $\alpha(4.2 \mathrm{~K})=192 \%$ ] (Refs. 30 and 31) and in contrast to a more moderate value of $\gamma=1.5[\alpha(\mathrm{RT})=125 \%$ and $\alpha(4.2 \mathrm{~K})$ $=185 \%]$ for the reference $\mathrm{Co}_{50} \mathrm{Fe}_{50}$ MTJs. Regarding the $T$ dependences of $R A_{\mathrm{AP}}$ and $R A_{\mathrm{P}}, R A_{\mathrm{AP}}$ decreased with increasing $T$, while $R A_{\mathrm{P}}$ was almost independent of $T$ for the CCFA MTJs [Fig. 8(b)]. These $T$ dependences of $R A_{\mathrm{AP}}$ and $R A_{\mathrm{AP}}$ were similar to those observed for $\mathrm{Co}_{70} \mathrm{Fe}_{30} / \mathrm{MgO} / \mathrm{Co}_{84} \mathrm{Fe}_{16}$ MTJs (Ref. 53) and CMS/MgO/ $\mathrm{Co}_{50} \mathrm{Fe}_{50}$ MTJs (Refs. 30 and 31). These results indicate that the decreasing TMR ratio with increasing $T$ for the CCFA MTJs was mainly due to the $R A_{\mathrm{AP}}$ decrease. To clarify the reason for the strong $T$ dependence of the TMR ratio, or equivalently that of the $R A_{\mathrm{AP}}$ observed for the CCFA/MgO/ $\mathrm{Co}_{50} \mathrm{Fe}_{50}$ MTJs, further systematic study is needed.

\section{CONCLUSION}

We fabricated fully epitaxial magnetic tunnel junctions (MTJs) with a full-Heusler alloy $\mathrm{Co}_{2} \mathrm{Cr}_{0.6} \mathrm{Fe}_{0.4} \mathrm{Al}$ (CCFA) thin film as a lower electrode, a $\mathrm{MgO}$ tunnel barrier, and a $\mathrm{Co}_{50} \mathrm{Fe}_{50}$ upper electrode. Pseudo-spin-valve-type, fully epi- taxial CCFA $/ \mathrm{MgO} / \mathrm{Co}_{50} \mathrm{Fe}_{50}$ MTJs with a CCFA film composition close to the stoichiometric one demonstrated high tunnel magnetoresistance (TMR) ratios of $90 \%$ at room temperature (RT) and $240 \%$ at $4.2 \mathrm{~K}$. These high TMR ratios were attributed to the increased spin polarization at the Fermi level, which was due to the increased ratio of the $B_{2}$ region to the $A_{2}$ region in CCFA films achieved by bringing the CCFA film composition close to the stoichiometric. Fully epitaxial CCFA/MgO/ $\mathrm{Co}_{50} \mathrm{Fe}_{50}$ MTJs with exchange biasing were also fabricated, where a $\mathrm{Co}_{50} \mathrm{Fe}_{50}$ upper electrode was used in a synthetic ferrimagnetic $\mathrm{Co}_{50} \mathrm{Fe}_{50} / \mathrm{Ru} / \mathrm{Co}_{90} \mathrm{Fe}_{10}$ trilayer exchange-iased with an $\mathrm{IrMn}$ layer through the $\mathrm{Co}_{90} \mathrm{Fe}_{10} / \mathrm{IrMn}$ interface. These exchange-biased CCFA $/ \mathrm{MgO} / \mathrm{Co}_{50} \mathrm{Fe}_{50}$ MTJs exhibited further enhanced TMR ratios of $109 \%$ at RT and $317 \%$ at $4.2 \mathrm{~K}$. We suggested enhancement of the TMR ratio by a coherent tunneling contribution for fully epitaxial CCFA/MgO/ $\mathrm{Co}_{50} \mathrm{Fe}_{50}$ MTJs.

These results confirm the promise of an epitaxial MTJ using a Co-based full-Heusler alloy as a key device structure for utilizing the potentially high spin polarization of this material system.

\section{ACKNOWLEDGMENTS}

The authors are grateful to Associate Professor Tetsuya Uemura, Associate Professor Masashi Arita, and Dr. Ken-ichi Matsuda for their helpful discussions and support. This work was partly supported by a Grant-in-Aid for Scientific Research (B) (Grant No. 18360143) and a Grant-in-Aid for Creative Scientific Research (Grant No. 14GS0301) from the Ministry of Education, Culture, Sports, Science and Technology, Japan. One of the authors (T.M.) was also supported by a Research Fellowship for Young Scientists from the Japan Society for the Promotion of Science.

${ }^{1}$ S. A. Wolf, D. D. Awschalom, R. A. Buhrman, J. M. Daughton, S. von Molnár, M. L. Roukes, A. Y. Chtchelkanova, and D. M. Treger, Science 294, 1488 (2001).

${ }^{2}$ R. A. de Groot, F. M. Mueller, P. G. van Engen, and K. H. J. Buschow, Phys. Rev. Lett. 50, 2024 (1983).

${ }^{3}$ T. Ambrose, J. J. Krebs, and G. A. Prinz, J. Appl. Phys. 87, 5463 (2000).

${ }^{4}$ B. Ravel, J. O. Cross, M. P. Raphael, V. G. Harris, R. Ramesh, and V. Saraf, Appl. Phys. Lett. 81, 2812 (2002).

${ }^{5}$ U. Geiersbach, A. Bergmann, and K. Westerholt, J. Magn. Magn. Mater. 240, 546 (2002).

${ }^{6}$ S. Kämmerer, S. Heitmann, D. Meyners, D. Sudfeld, A. Thomas, A. Hütten, and G. Reiss, J. Appl. Phys. 93, 7945 (2003).

${ }^{7}$ L. J. Singh, Z. H. Barber, Y. Miyoshi, Y. Bugoslavsky, W. R. Brandford, and L. F. Cohen, Appl. Phys. Lett. 84, 2367 (2004).

${ }^{8}$ R. Kelekar and B. M. Clemens, J. Appl. Phys. 96, 540 (2004).

${ }^{9}$ A. Hirohata, H. Kurebayashi, S. Okamura, M. Kikuchi, T. Masaki, T. Nozaki, N. Tezuka, and K. Inomata, J. Appl. Phys. 97, 103714 (2005).

${ }^{10}$ K.-i. Matsuda, T. Kasahara, T. Marukame, T. Uemura, and M. Yamamoto, J. Cryst. Growth 286, 389 (2006).

${ }^{11}$ T. Ishikawa, T. Marukame, K.-i. Matsuda, T. Uemura, M. Arita, and M. Yamamoto, J. Appl. Phys. 99, 08J110 (2006).

${ }^{12}$ H. Kijima, T. Ishikawa, T. Marukame, H. Koyama, K. Matsuda, T. Uemura, and M. Yamamoto, IEEE Trans. Magn. 42, 2688 (2006).

${ }^{13}$ S. Ishida, S. Fujii, S. Kashiwagi, and S. Asano, J. Phys. Soc. Jpn. 64, 2152 (1995).

${ }^{14}$ S. Picozzi, A. Continenza, and A. J. Freeman, Phys. Rev. B 66, 094421 (2002).

${ }^{15}$ P. J. Webster, J. Phys. Chem. Solids 32, 1221 (1971).

${ }^{16}$ K. Inomata, S. Okamura, R. Goto, and N. Tezuka, Jpn. J. Appl. Phys., Part 2 42, L419 (2003).

${ }^{17}$ S. Kämmerer, A. Thomas, A. Hütten, and G. Reiss, Appl. Phys. Lett. 85, 
79 (2004)

${ }^{18}$ H. Kubota, J. Nakata, M. Oogane, Y. Ando, A. Sakuma, and T. Miyazaki, Jpn. J. Appl. Phys., Part 2 43, L984 (2004).

${ }^{19}$ Y. Sakuraba, J. Nakata, M. Oogane, H. Kubota, Y. Ando, A. Sakuma, and T. Miyazaki, Jpn. J. Appl. Phys., Part 2 44, L1100 (2005).

${ }^{20}$ S. Okamura, A. Miyazaki, S. Sugimoto, N. Tezuka, and K. Inomata, Appl Phys. Lett. 86, 232503 (2005).

${ }^{21}$ T. Marukame, T. Kasahara, K.-i. Matsuda, T. Uemura, and M. Yamamoto, Jpn. J. Appl. Phys., Part 2 44, L521 (2005).

${ }^{22}$ T. Marukame, T. Kasahara, K.-i. Matsuda, T. Uemura, and M. Yamamoto, IEEE Trans. Magn. 41, 2603 (2005)

${ }^{23}$ Y. Sakuraba, J. Nakata, M. Oogane, Y. Ando, H. Kato, A. Sakuma, T. Miyazaki, and H. Kubota, Appl. Phys. Lett. 88, 022503 (2006).

${ }^{24}$ Y. Sakuraba, M. Hattori, M. Oogane, Y. Ando, H. Kato, A. Sakuma, T. Miyazaki, and H. Kubota, Appl. Phys. Lett. 88, 192508 (2006).

${ }^{25}$ K. Inomata, S. Okamura, A. Miyazaki, M. Kikuchi, N. Tezuka, M. Wojcik, and E. Jedryka, J. Phys. D 39, 816 (2006)

${ }^{26} \mathrm{M}$. Yamamoto, T. Marukame, T. Ishikawa, K. Matsuda, T. Uemura, and M. Arita, J. Phys. D 39, 824 (2006)

${ }^{27}$ T. Marukame, T. Ishikawa, K.-i. Matsuda, T. Uemura, and M. Yamamoto, J. Appl. Phys. 99, 08A904 (2006).

${ }^{28}$ T. Marukame, T. Ishikawa, K.-i. Matsuda, T. Uemura, and M. Yamamoto, Appl. Phys. Lett. 88, 262503 (2006).

${ }^{29}$ T. Marukame, T. Kasahara, K.-i. Matsuda, T. Uemura, and M. Yamamoto, IEEE Trans. Magn. 42, 2652 (2006).

${ }^{30}$ T. Ishikawa, T. Marukame, H. Kijima, K.-i. Matsuda, T. Uemura, M. Arita, and M. Yamamoto, Appl. Phys. Lett. 89, 192505 (2006).

${ }^{31}$ T. Marukame, H. Kijima, T. Ishikawa, K.-i. Matsuda, T. Uemura, and M. Yamamoto, J. Magn. Magn. Mater. 310, 1946 (2007).

${ }^{32}$ H. Kijima, T. Ishikawa, T. Marukame, K.-i. Matsuda, T. Uemura, and M. Yamamoto, J. Magn. Magn. Mater. 310, 2006 (2007).
${ }^{33}$ T. Marukame, T. Ishikawa, S. Hakamata, K.-i. Matsuda, T. Uemura, and M. Yamamoto, Appl. Phys. Lett. 90, 012508 (2007).

${ }^{34}$ R. Kelekar and B. M. Clemens, Appl. Phys. Lett. 86, 232501 (2005).

${ }^{35} \mathrm{~K}$. Yakushiji, K. Saito, S. Mitani, K. Takanashi, Y. K. Takahashi, and K. Hono, Appl. Phys. Lett. 88, 222504 (2006).

${ }^{36}$ X. Y. Dong et al., Appl. Phys. Lett. 86, 102107 (2005).

${ }^{37}$ M. C. Hickey et al., Appl. Phys. Lett. 86, 252106 (2005).

${ }^{38}$ Y. Miura, K. Nagao, and M. Shirai, Phys. Rev. B 69, 144413 (2004).

${ }^{39}$ T. Block, C. Felser, G. Jakob, J. Ensling, B. Mühling, P. Gütlich, and R. J. Cava, J. Solid State Chem. 176, 646 (2003).

${ }^{40}$ C. Felser et al., J. Phys.: Condens. Matter 15, 7019 (2003).

${ }^{41}$ K. Kobayashi, R. Y. Umetsu, R. Kainuma, K. Ishida, T. Oyamada, A. Fujita, and K. Fukamichi, Appl. Phys. Lett. 85, 4684 (2004).

${ }^{42}$ S. Picozzi, A. Continenza, and A. J. Freeman, Phys. Rev. B 69, 094423 (2004).

${ }^{43}$ S. S. P. Parkin et al., J. Appl. Phys. 85, 5828 (1999).

${ }^{44}$ W. C. Ellis and E. S. Greiner, Trans. Am. Soc. Met. 29, 415 (1941).

${ }^{45}$ T. Ishikawa, T. Marukame, K.-i. Matsuda, T. Uemura, and M. Yamamoto, IEEE Trans. Magn. 42, 3002 (2006)

${ }^{46}$ J. G. Simmons, J. Appl. Phys. 34, 1793 (1963).

${ }^{47}$ S. Yuasa, T. Nagahama, A. Fukushima, Y. Suzuki, and K. Ando, Nat. Mater. 3, 868 (2004)

${ }^{48}$ M. Jullière, Phys. Lett. 54, 225 (1975).

${ }^{49}$ J. Mathon and A. Umerski, Phys. Rev. B 60, 1117 (1999).

${ }^{50}$ D. J. Mosma and S. S. P. Parkin, Appl. Phys. Lett. 77, 720 (2000).

${ }^{51}$ W. H. Butler, X.-G. Zhang, T. C. Schulthess, and J. M. Maclaren, Phys. Rev. B 63, 054416 (2001).

${ }^{52}$ J. Mathon and A. Umerski, Phys. Rev. B 63, 220403R (2001).

${ }^{53}$ S. S. P. Parkin, C. Kaiser, A. Panchula, P. M. Rice, B. Hughes, M. Samant, and S.-H. Yang, Nat. Mater. 3, 862 (2004). 